\title{
Stable Multiwavelength Semiconductor Laser Using FWM and SBS-Assisted Filter
}

\author{
C. H. Yeh, Member, IEEE, C. W. Chow, Member, IEEE, Y. F. Wu, Student Member, IEEE, \\ F. Y. Shih, Student Member, IEEE, J. H. Chen, Member, IEEE, and C. L. Pan, Senior Member, IEEE
}

\begin{abstract}
A stable and flattened quadruple-wavelength laser system is proposed and experimentally investigated. The laser consists of an external injection Fabry-Pérot laser diode (FP-LD) and a stimulated-Brillouin-scattering (SBS)-assisted filter. In the measurement, the power difference $(\Delta P)$ of the lasing quadruple-wavelength can be enhanced within $2.0 \mathrm{~dB}$ by SBS-assisted filtering after $22-\mathrm{km}$ single-mode fiber (SMF) propagation. In addition, we can change the two injected wavelengths for tuning the quadruple-wavelength. Besides, the output stability of the proposed laser has been studied and analyzed.
\end{abstract}

Index Terms-Fabry-Pérot laser diode (FP-LD), four-wave mixing (FWM), stimulated Brillouin scattering (SBS).

\section{INTRODUCTION}

$\mathbf{R}$ ECENTLY, stable and tunable erbium-doped fiber (EDF) ring lasers with single- or multiwavelength operations are desirable for wavelength division multiplexed (WDM) transmission systems, optical device testing, fiber-optic sensor systems, and precise spectroscopy. However, owing to the homogeneous gain broadening characteristic of the EDF, the maximum number of generated lasing wavelength could only be two or three [1]. Thus, to solve the limitation by the homogeneous broadening of EDF at room temperature, several techniques have been proposed and investigated [2]-[6]. Furthermore, to improve the stability of the multiwavelength EDF lasers, many different techniques for reducing mode competition have been proposed and investigated, such as cooling the EDF at $77 \mathrm{~K}$ [7], introducing active overlapping linear cavities [8], or using the polarization hole-burning [6].

In this study, we propose and experimentally investigate an external-injection Fabry-Pérot laser diode (FP-LD) to achieve the flattened quadruple-wavelength output by using four-wave mixing (FWM) and stimulated-Brillouin-scattering (SBS)-assisted filter. Here, using two external-injected wavelengths into the FP-LD can achieve dual-wavelength lasing and produce two sidemodes due to the FWM. Then, the SBS-assisted filter can

Manuscript received February 26, 2011; revised August 02, 2011; accepted August 14, 2011. Date of current version October 19, 2011.

C. H. Yeh, Y. F. Wu, and F. Y. Shih are with the Information and Communications Research Laboratories, Industrial Technology Research Institute (ITRI), Hsinchu 31040, Taiwan (e-mail: yeh1974@gmail.com).

C. W. Chow is with the Department of Photonics and Institute of ElectroOptical Engineering, National Chiao Tung University, Hsinchu 30010, Taiwan.

J. H. Chen is with the Department of Photonics, Feng Chia University, Taichung 40724, Taiwan.

C. L. Pan is with the Department of Physics and Institute of Photonics Technologies, National Tsing Hua University, Hsinchu 30013, Taiwan.

Color versions of one or more of the figures in this letter are available online at http://ieeexplore.ieee.org.

Digital Object Identifier 10.1109/LPT.2011.2165836 be used to suppress the output powers of dual-wavelength to $20 \mathrm{~dB}$ after $22 \mathrm{~km}$ single-mode fiber (SMF) transmission. In addition, we can change the two injected-wavelengths for the quadruple-wavelength tuning in the $C$-band. The experimental results show that the proposed laser not only presents the flattened quadruple-wavelength output with wavelength tunabiltiy, but also has good output stability.

\section{EXPERIMENT AND RESULTS}

Fig. 1 shows the experimental setup of the proposed quadruple-wavelength laser configuration. The proposed laser scheme was constructed by a FP-LD, two distributed feedback laser diodes (DFB-LDs), an optical circulator (OC), a polarization controller $\left(\mathrm{PC}_{1}\right)$, and a $1 \times 2$ and 50:50 optical coupler (CP). Here, the mode-spacing and threshold current of multilongitudinal-mode (MLM) FP-LD were $1.11 \mathrm{~nm}$ and $9.5 \mathrm{~mA}$, respectively. In the experiment, the bias current of the FP-LD was operated at $25 \mathrm{~mA}$ under the temperature of $25^{\circ} \mathrm{C}$. Fig. 2(a) presents the output spectrum of the MLM FP-LD before optical-injection, and the power of the center wavelength was about $-5 \mathrm{dBm}$. The optical spectrum was measured by an optical spectrum analyzer (OSA) with a resolution of $0.05 \mathrm{~nm}$. Two DFB-LDs were connected to the CP, OC and PC, and then to the FP-LD, as shown in Fig. 1. The wavelengths of the two DFB-LDs were 1545.10 and $1546.21 \mathrm{~nm}$. Their output powers and linewidth were $-2 \mathrm{dBm}$ and $\sim 100 \mathrm{kHz}$ respectively. The total insertion losses were: $\mathrm{CP}(\sim 3.2 \mathrm{~dB})$, OC $(\sim 0.7 \mathrm{~dB})$ and $\mathrm{PC}(\sim 1.2 \mathrm{~dB})$. Therefore, the actually continuous-wave $(\mathrm{CW})$ injected power into the FP-LD was nearly $-7 \mathrm{dBm}$ after passing through these three passive components. The two $\mathrm{CW}$ injection lights could be regarded as the pump lights for the FWM. The $\mathrm{PC}_{1}$ in the experiment could be used to adjust the proper polarization to maximum the effect of FWM. In the experiment, the FP-LD (the master laser) is temperature stabilized. By adjusting the wavelengths and the polarizations of the two pumps, FWM can be observed at the output port of the FP-LD. When the phase matching condition of the injected pump signals are satisfied, FWM can be observed in semiconductor materials such as in semiconductor optical amplifier (SOA) and FP-LD [9]. Detail discussion has also been reported in [9].

Fig. 2(b) shows the output spectrum of dual-wavelength in the proposed laser scheme when the two $\mathrm{CW}$ lights were used to inject into FP-LD, observed at point "a" in Fig. 1. Due to the FWM effect, two sidemodes were also produced at 1543.98 and $1547.34 \mathrm{~nm}$ with -28.5 and $-30.7 \mathrm{dBm}$ peak powers, respectively. Hence, four wavelengths of the proposed quadruple- 


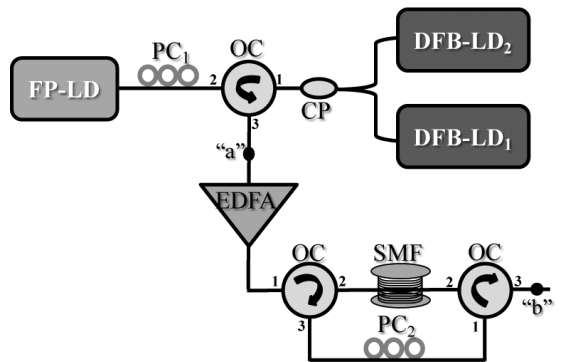

Fig. 1. Experimental setup of proposed quadruple-wavelength laser configuration.
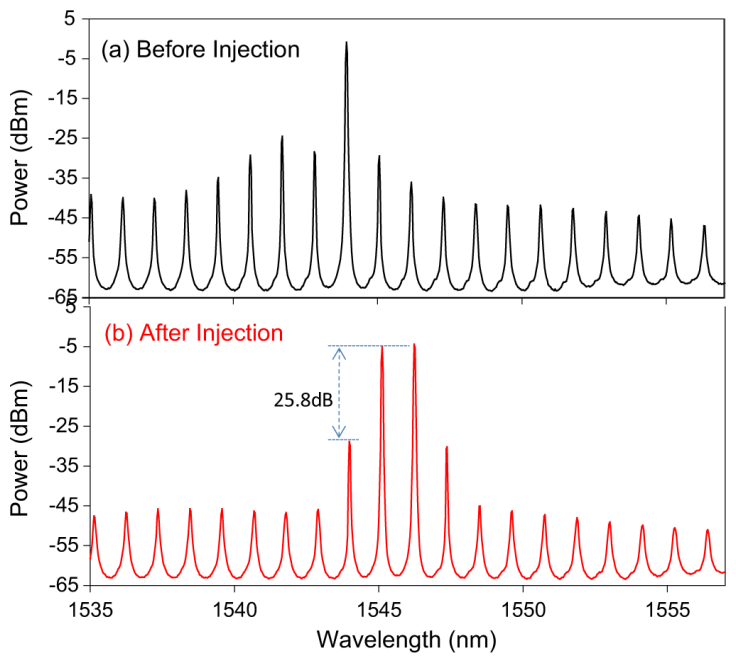

Fig. 2. Output spectrum of MLM FP-LD (a) before and (b) after optical-injection when the FP-LD is operated at $25 \mathrm{~mA}$ under the temperature of $25^{\circ} \mathrm{C}$.

wavelength laser scheme can be obtained by the external-injection mechanism. However, in this preliminary measurement result (without using the SBS-assisted filter), the power variation $(\Delta P)$ of the observed quadruple-wavelength is $25.8 \mathrm{~dB}$.

Then, in order to achieve the flattened output of the quadruple-wavelength, the method used in the proposed laser system was the SSB-assisted filter [10], which consisted of two OCs, a $\mathrm{PC}\left(\mathrm{PC}_{2}\right)$ and a spool of single-mode fiber (SMF). The SSB-assisted filter was used to serve as a wavelength-independent narrow band notch filter [10]. The suppression ratio was determined by the power of the input regardless of the wavelength. The suppression ratio could increase significantly when the power of input wavelength was increased. The SBS-assisted filter can suppress the two lasing wavelengths of higher output power while keeping the powers of the two sidemodes. Besides, we can also adjust the $\mathrm{PC}_{2}$ to maximize the suppression ratio of the pumped dual-wavelength by SBS-assisted filter. In the experiment, an erbium-doped fiber amplifier (EDFA) with $16 \mathrm{dBm}$ saturation power was used to amplify the laser output to generate the SBS in the SMF for the SBS-assisted filtering, as shown in Fig. 1. However, the EDFA used will also increase the background amplified spontaneous emission (ASE) in the experiment.

Different distances of SMF were used in the measurement $(4,8,10,12,15,18,20,22,24$, and $30 \mathrm{~km})$ to study the suppression ratio performance. Figs. 3(a) to 3(d) show the output spectra of the proposed quadruple-wavelength laser, observed at point "b" in Fig. 1, when the SMF distances are
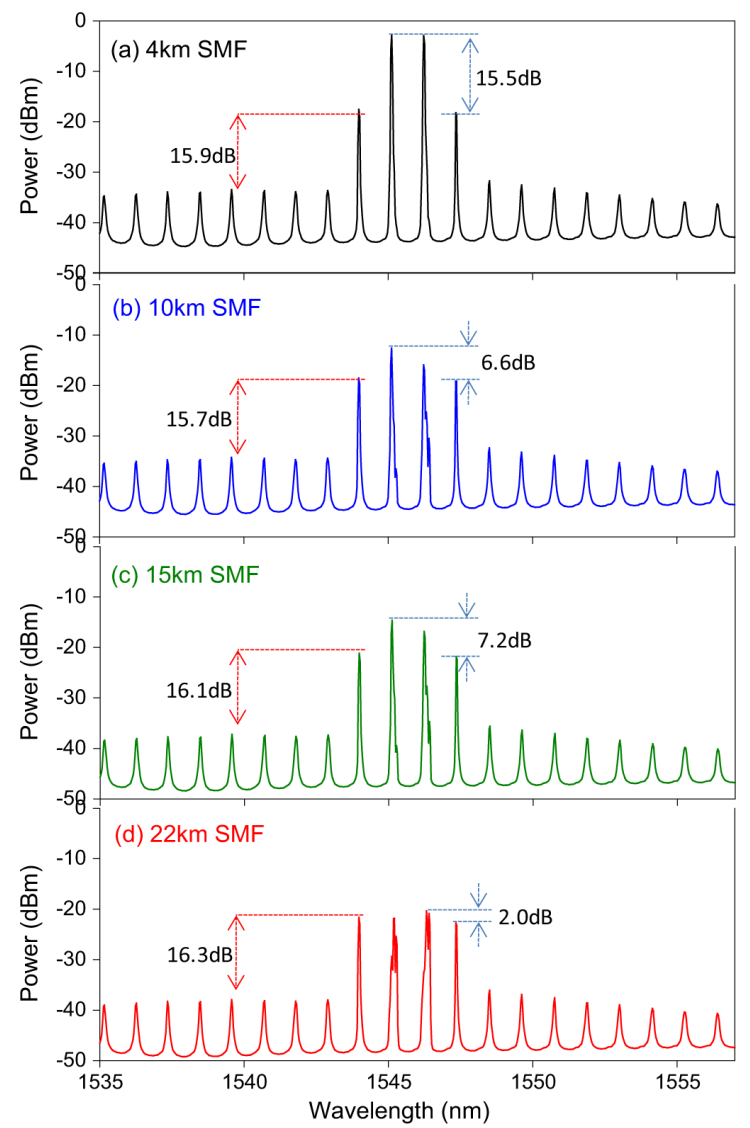

Fig. 3. Output spectra of the proposed quadruple-wavelength laser architecture, observing at "b" point in Fig. 1, when the SMF distances are (a) 4, (b) 10, (c) 15 , and (d) $22 \mathrm{~km}$, respectively.

4, 10, 15 and $22 \mathrm{~km}$ respectively. According to the experimental results, when the SMF length is increased gradually, the suppression ratio of pumped dual-wavelength can be also suppressed. Hence, the output power variations of the lasing quadruple-wavelength can be measured within 15.5, 6.5, 7.2 and $2.0 \mathrm{~dB}$, respectively, as shown in Figs. 3(a) to 3(d). And, the measured quadruple-wavelength are $1543.98,1545.12,1546.23$ and $1547.37 \mathrm{~nm}$ with $-21.6,-21.9,-20.3$ and $-22.3 \mathrm{dBm}$ peak powers, respectively, when the SMF length is $22 \mathrm{~km}$ long in the experiment. Besides, when the SMF length is increased gradually, the background ASE spectrum is also reduce due to the SBS-assisted filtering, as also shown in Figs. 3(a) to 3(d). The two pump light sources (from DFB-LDs) and the FWM generated two idle lights are amplified and launched into the SBS-assisted filter via the left OC. The powers of the two pumps are above the threshold of the SBS. Thus, Stokes signals could only be generated by the pumps. As shown in Fig. 1, the Stokes signals are relaunched into the SMF via the right OC in a direction opposite to the pumps. This will cause induced acoustic grating [11], enhancing more backscattering of pumps into the Stokes signals, thus further suppressing the pumps [11].

Fig. 4 also shows the power variations of the proposed quadruple-wavelength laser configuration at the SMF lengths from 4 to $30 \mathrm{~km}$ in two measurements. In Fig. 4, when the SMF length is increased gradually, the power difference decreases. However, we can also observe two dropping points while the SMF lengths are 10 and $22 \mathrm{~km}$, respectively, and 


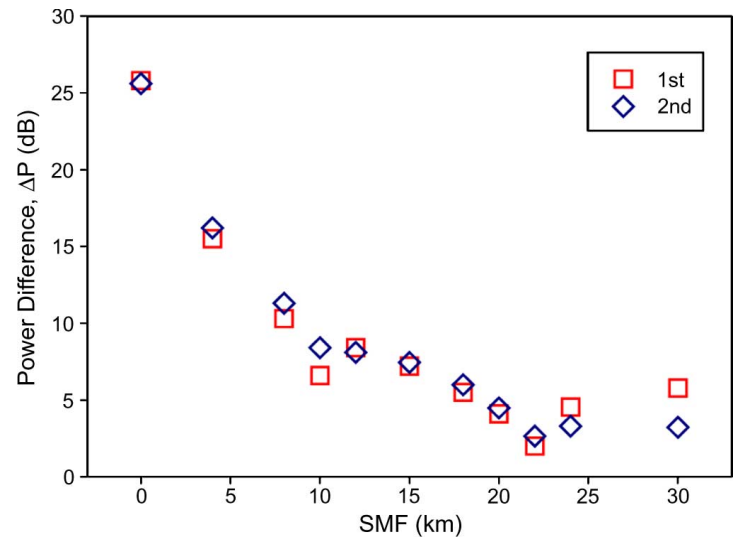

Fig. 4. Measured power variations of the proposed quadruple-wavelength laser configuration at the SMF distances of 4 to $30 \mathrm{~km}$.

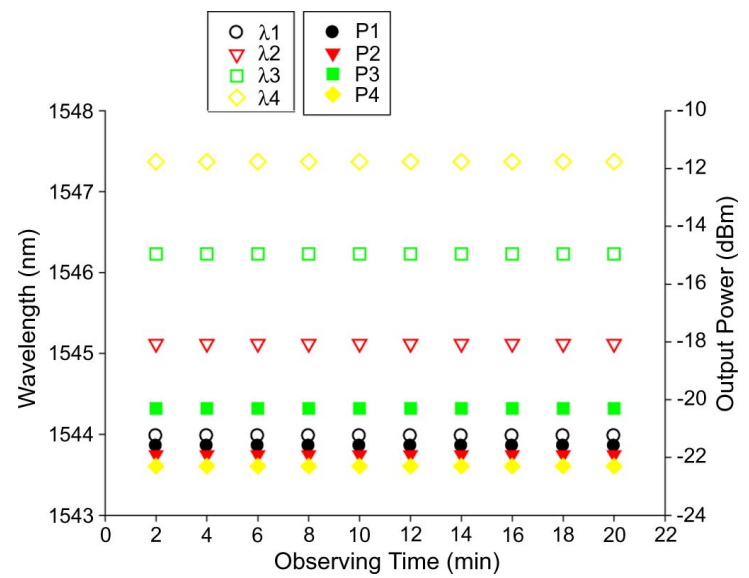

Fig. 5. Output stabilities of power and wavelength for the proposed quadruplewavelength laser scheme over 20 min observing-time.

their corresponding power differences are 6.6 and $2.0 \mathrm{~dB}$ at the first measurement. We believe that the two dropping points are the measurement errors. As a result, we can obtain the better quadruple-wavelength output with the $\Delta P$ of $2.0 \mathrm{~dB}$ when the SMF of $22 \mathrm{~km}$ long is used in the SBS-assisted filter. Mode partition noise is caused by the power fluctuation among longitudinal modes. As the two optical pump sources are very stable light sources produced by tunable lasers; and the FWM can only be generated when the two optical pumps should satisfy the phase and polarization matching conditions. We believe that the mode partition noise will not be enhanced.

To investigate the output power and wavelength stabilities of the four lasing wavelengths in the proposed laser, a short-term stability test in 20 mins observation time was performed. As shown in Fig. 5, the power and wavelength variations of the four lasing wavelengths of the proposed laser are negligible.

The proposed quadruple-wavelength can be also tuned when the two pumped wavelengths are changed. Fig. 6 shows the optical spectra of the laser output when the two injected wavelengths are set at 1538.5 and $1539.6 \mathrm{~nm} ; 1541.8$ and $1542.9 \mathrm{~nm}$; 1545.1 and $1546.2 \mathrm{~nm}$, and 1548.5 and $1549.6 \mathrm{~nm}$, respectively. The results show that four stable laser output can be successfully obtained.

\section{CONCLUSION}

We have proposed and experimentally investigated an optical injected FP-LD to achieve the flattened quadruple-wave-

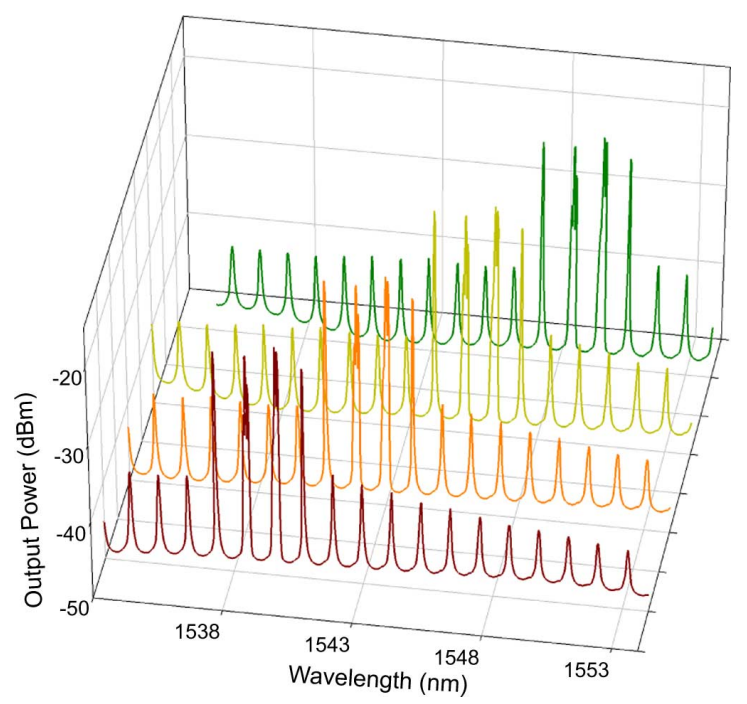

Fig. 6. Output spectra of the proposed laser scheme when the two injected wavelengths are set at 1538.5 and $1539.6 \mathrm{~nm}, 1541.8$ and $1542.9 \mathrm{~nm}, 1545.1$ and $1546.2 \mathrm{~nm}$, and 1548.5 and $1549.6 \mathrm{~nm}$, respectively, for quadruple-wavelength tuning.

length output by using FWM and SBS-assisted filter. Employing two external-injected wavelengths into the FP-LD can generate dual-wavelength lasing and also produce two sidemodes due to the FWM. Here, the SBS-assisted filter can be used to suppress the output powers of two pumped wavelengths via $22 \mathrm{~km}$ SMF transmission. The proposed laser scheme not only presents the flattened quadruple-wavelength output with wavelength tunability, but also has good output stability.

\section{REFERENCES}

[1] C.-H. Yeh, M.-C. Lin, C.-C. Lee, and S. Chi, "Fiber Bragg grating-based multiplexed sensing system employing fiber laser scheme with semiconductor optical amplifier," Jpn. J. Appl. Phys., vol. 44, pp. 6590-6592, 2005.

[2] J. N. Maran, S. LaRochelle, and P. Besnard, "Erbium-doped fiber laser simultaneously mode locked on more than 24 wavelengths at room temperature," Opt. Lett., vol. 28, pp. 2082-2084, 2003.

[3] G. Das and J. Lit, "Wavelength switching of a fiber laser with a Sagnac loop reflector," IEEE Photon. Technol. Lett., vol. 16, no. 1, pp. 60-62, Jan. 2004.

[4] C. S. Kim, F. Farokhrooz, and J. Kang, "Electro-optic wavelength-tunable fiber ring laser based on cascaded composite sagnac loop filters," Opt. Lett., vol. 29, pp. 1677-1679, 2004.

[5] T. Miyazaki, N. Edagawa, S. Yamamoto, and S. Akiba, "A multiwavelength fiber ring-laser employing a pair of silica-based arrayed-waveguide- gratings," IEEE Photon. Technol. Lett., vol. 9, no. 7, pp. 910-912, Jul. 1997.

[6] Y. G. Liu, X. Feng, and S. Yuan, "Simultaneous four-wavelength lasing oscillations in an erbium-doped fiber laser with two high birefringence fiber Bragg gratings," Opt. Express, vol. 12, pp. 2056-2061, 2004.

[7] S. Yamashita and K. Hotate, "Multiwavelength erbium-doped fiber laser using intracavity etalon and cooled by liquid nitrogen," Electron. Lett., vol. 32, pp. 1298-1299, 1996.

[8] Q. H. Mao and J. Lit, "Multiwavelength erbium-doped fiber lasers with active overlapping linear cavities," J. Lightw. Technol., vol. 21, no. 1, pp. 160-169, Jan. 2003.

[9] R. Nietzke, P. Panknin, W. Elsasser, and E. O. Gobel, "Four-wave mixing in GaAs/AlGaAs semiconductor lasers," IEEE J. Quantum Electron., vol. 25, no. 6, pp. 1399-1406, Jun. 1989.

[10] S. H. Lee and S. H. Kim, "All optical gain-clamping in erbium-doped fiber amplifier using stimulated Brillouin scattering," IEEE Photon. Technol. Lett., vol. 10, no. 9, pp. 1316-1318, Sep. 1998.

[11] W. Li and J. Yao, "Microwave and Terahertz generation based on photonically assisted microwave frequency twelvetupling with large tenability," IEEE Photon. J., vol. 2, no. 6, pp. 954-959, Dec. 2010. 